\title{
L'interdisciplinarité : un alibi ou un ferment dans la réforme de la recherche?
}

NSS a régulièrement pris position, au travers d'éditoriaux, sur la situation institutionnelle de la recherche. Il s'agissait pour nous de prendre part à un débat dans lequel nous étions plus particulièrement attentifs aux conditions d'exercice de l'activité scientifique du point de vue des pratiques interdisciplinaires. Ainsi nous sommesnous demandé "Quelle recherche voulons-nous? De quelle recherche avons-nous besoin? » (NSS, 14, 3) ou bien avons-nous rappelé notre projet éditorial «Natures Sciences Sociétés : l'actualité d'une ambition éditoriale» (NSS 14,1$)$ ou bien avons-nous pris position par rapport à la recherche dite de "mode 2 » $(N S S, 12,1)$ en ce qu'elle nous semblait refléter davantage les situations concrètes du travail interdisciplinaire.

Deux raisons plaident pour revenir à nouveau sur ces questions et réaffirmer les objectifs de la revue. Tout d'abord, les profonds bouleversements en cours, ou attendus, qui tendent à transformer les modes de travail jusqu'au métier de chercheur et d'enseignant lui-même. En second lieu, la mise en place de critères d'évaluation de l'activité scientifique, et plus particulièrement le récent classement AERES des revues.

Les établissements publics de recherche, CNRS en tête, sont donc conviés à se doter d'une nouvelle mission d'agence de moyens, en plus de celle d'opérateur de recherche qui était jusqu'à présent au cœur de leur activité. Sur le plan budgétaire, cette nouvelle mission est appelée à devenir rapidement majoritaire. Le CNRS n'entend exercer sa mission d'opérateur de recherche que pour une partie des unités mixtes de recherche dont il a aujourd'hui la tutelle - unités dites «stratégiques » qui ne représenteraient que $50 \%$ de l'effectif actuel dont on veut extirper les « unités de complaisance »-, offrant en particulier aux universités, nouvel opérateur de recherche grâce à la loi LRU, les unités restantes qualifiées de «non stratégiques ». Par ailleurs, il n'a échappé à personne que la création des instituts au CNRS a été particulièrement difficile pour deux communautés scientifiques qui partagent une forte présence au sein de notre production éditoriale, les Sciences humaines et sociales ainsi que les Sciences de la vie. Enfin, la création de « chaires mixtes universités-organismes » offre des ponts inédits entre la recherche et l'enseignement, au prix semble-t-il d'une diminution des postes traditionnellement mis au concours.

Ces différents traits d'une réforme en cours plaident-ils pour une recherche favorisant les échanges et les circulations entre les diverses communautés scientifiques? Certes, l'interdisciplinarité en est l'un des maîtres mots, jusqu'à vouloir privilégier au CNRS les recrutements par des commissions interdisciplinaires. Mais est-elle compatible avec l'élitisme et la précarité qui semblent au cœur de cette réorganisation institutionnelle? Il est en effet difficile de ne pas voir dans la distinction des unités «stratégiques » ou «non stratégiques » un principe de hiérarchie de même que l'attribution des chaires mixtes se fera sur la base d'une stricte excellence scientifique privilégiant des individualités. Si l'on peut également craindre une certaine précarité, c'est que, sous prétexte que l'on n'est "pas chercheur à vie » ou pour cause de mobilité, objectifs louables en soi, on veut privilégier la recherche sur projet, en espérant de ces communautés éphémères qu'elles soient moins routinières que celles constituées sur la base d'une unité de recherche et plus aptes à l'opérationnalisation propre au court terme. Sans aller jusqu'à l'opposition franche de l'une et de l'autre, la réforme jette le soupçon sur un certain type d'accumulation de connaissances fait de travail d'équipes et d'investissement intellectuel inscrit dans la durée comme c'est le cas dans un laboratoire.

Les conditions d'exercice des pratiques interdisciplinaires supposent bien entendu que se tissent des réseaux entre communautés épistémiques selon une logique de projet. Mais, paradoxalement, les traits majeurs de cette réforme sont susceptibles de les fragiliser. 
Il faut reconnaître que, forts de leur interdisciplinarité structurelle, les établissements de recherche se sont révélés plus aptes à construire des transversalités entre disciplines au travers de programmations affranchies du carcan disciplinaire. À l'inverse, les universités apparaissent souvent prisonnières d'un enseignement académique privilégiant la frontière disciplinaire, même si des efforts réels ont été faits en ce sens au niveau des masters, souvent à l'initiative d'enseignants-chercheurs convaincus, ainsi que l'ont montré les journées de l'association NSS Dialogues en février 2007 sur « les formations interdisciplinaires » (NSS, vol. 16, supplément 2008).

L'excellence scientifique, revendiquée comme l'un des objectifs de la réforme, est le produit d'un certain type d'évaluation dont on sait combien les critères qui la fondent ne rendent pas compte des recherches à vocation interdisciplinaire. Ces dernières brouillent les repères habituels d'une évaluation basée sur des publications hautement spécialisées et privilégiant l'excellence reconnue dans la discipline. NSS s'est souvent interrogée sur les freins de ce type d'évaluation, que ce soit au niveau des publications ou à celui des carrières individuelles, et c'est précisément pour les dépasser que la revue fut fondée avec l'ambition de conjuguer l'excellence scientifique, même d'un point de vue disciplinaire, et la recherche en situation d'interdisciplinarité. Dans une telle situation, il est précisément difficile de privilégier tel ou tel individu car les pratiques interdisciplinaires sont particulièrement exigeantes sur le plan collectif, nécessitant une abnégation, une écoute, une modestie même qui font du travail d'équipe, de son histoire une marque particulière de la démarche collective et de la production scientifique qui en résulte.

La précarité, autre dimension de la réforme sousjacente aux nouvelles modalités de recrutement ou de gestion des carrières, s'accommode mal de ce type d'entreprise car l'accumulation de connaissances dans un tel contexte demande du temps et nombre de projets interdisciplinaires sont inscrits dans une temporalité qui va au-delà du format habituel de la production des connaissances par projet. Ce n'est pas un hasard si la notion d'observatoire a été particulièrement réfléchie dans les équipes engagées - au sens fort du terme - sur la voie de l'interdisciplinarité. Ils représentent un format organisationnel susceptible d'assurer dans de bonnes conditions l'évaluation des programmes et d'accompagner les dynamiques intellectuelles propres à la confrontation et au partage de concepts disciplinaires. Ils remplissent par ailleurs les conditions pour la mise en œuvre de recherches menées avec des partenaires étrangers au monde académique.

La réforme en cours concerne également les revues. Récemment, l'AERES a proposé un classement des revues scientifiques, selon une notation $\mathrm{A}, \mathrm{B}$, ces deux premières notes étant assimilées à une publication dans une « revue à comité de lecture », la troisième note, $\mathrm{C}$, étant particulièrement stigmatisante. NSS a été classée en B. Outre le fait que la procédure aboutissant à un tel classement est d'une opacité totale, qu'elle n'a donné lieu à aucun dialogue entre évaluateurs et évalués, condition minimale pour échapper à une évaluation comptable et «bibliométrique » et restituer la spécificité d'un projet éditorial, ce classement reflète le difficile ajustement entre les intentions affichées de promotion de l'interdisciplinarité dans les organismes de recherche et la reconnaissance concrète des communautés acceptant d'en faire le pari. De ce point de vue, NSS partage les réactions soulevées dans les communautés éditoriales par rapport à un tel classement.

NSS a la chance d'être soutenue par six organismes de recherche qui lui reconnaissent ainsi un rôle majeur dans la structuration des équipes qui ont fait le choix de l'interdisciplinarité au nom de l'exigence scientifique elle-même. Souhaitons qu'un tel engagement soit reconnu dans le nouveau paysage de la recherche que la réforme en cours dessine. Le dossier «Interdisciplinarité » que nous avons ouvert voici quatre ans $(N S S, 12,1)$ et qui donne lieu à des contributions régulières, comme c'est le cas dans ce numéro, est une invite pour nos lecteurs à entrer dans le débat sur une question qui est au cœur de l'actualité de la réforme engagée : à quelles conditions - institutionnelles, organisationnelles, épistémiques - l'interdisciplinarité peut-elle représenter un projet scientifique de portée générale? C'est la question que nous devons nous poser si nous voulons que la réforme en cours fasse de l'ambition interdisciplinaire qu'elle affiche autre chose qu'un alibi, un réel ferment pour la transformation de l'activité scientifique. 